
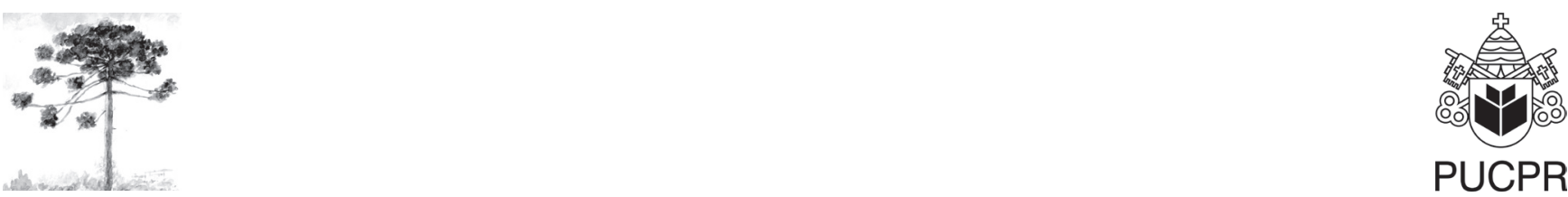

PUCPR

\title{
VARIAÇÃO ANUAL DA BIOMASSA DE Telebasis (ODONATA: COENAGRIONIDAE), JUNTO À Eichbornia azurea (SW.) KUN- TH, NA LAGOA DO CAMARGO (LATERAL AO RIO PARA- NAPANEMA, SÃO PAULO)
}

\author{
Annual variation of Telebasis (Insecta) biomass, with Eichhornia azurea (Sw.) \\ Kunth in Camargo Lake (on the side of Paranapanema River, São Paulo)
}

\author{
João Ânderson Fulan ${ }^{[a]}$, Raoul Henry ${ }^{[b]}$
}

[a] Doutor em Ciências Biológicas (Zoologia) pela Universidade Estadual Paulista Júlio de Mesquita Filho, professor adjunto I da Universidade Federal do Amazonas (UFAM), Humaitá, AM - Brasil, e-mails: joaofulan@ig.com.br

[b] Doutorado em Ecologia e Recursos Naturais pela Universidade Federal de São Carlos (UFSCar), professor titular da Universidade Estadual Paulista Júlio de Mesquita Filho, Botucatu, SP - Brasil, e-mails: rhenry@ibb.unesp.br

\section{Resumo}

O objetivo deste trabalho foi estimar a variação da biomassa das ninfas de Telebasis associadas à Eichhornia azurea, na Lagoa do Camargo, lateral ao Rio Paranapanema, São Paulo, durante o período de março de 2004 a março de 2005. As ninfas de Telebasis foram removidas da macrófita por lavagem em baldes contendo, respectivamente, formol 8\%, 4\% e água. O comprimento total de cada ninfa foi medido e sua biomassa calculada a partir de seu peso seco após 24 horas em estufa. A relação entre o comprimento total $(\mathrm{mm})$ e o peso seco $(\mathrm{mg})$ de cada imaturo de Telebasis mostrou uma curva exponencial de ajuste com equação y = 0,31 e 0,36x e R2 = 0,96. Em conclusão, houve um aumento na biomassa de Telebasis no período seco, em virtude do aumento na oferta de alimento provocado pela redução no nível da água. A biomassa foi menor nos meses mais frios possivelmente pela emergência dos adultos, sendo registrados apenas indivíduos jovens e com menor biomassa.

Palavras-chave: Biomassa. Macrófitas. Odonatos. Telebasis.

\section{Abstract}

The aim of this work was to estimate the biomass variation of Telebasis nymphs, living on roots of Eichhornia azurea in Camargo Lake, a lateral lake connected with Paranapanema River (São 
Paulo), from March 2004 to March 2005. The Telebasis nymphs were removed from the aquatic macrophytes by plant washing in buckets containing $8 \%$ and $4 \%$ formaldehyde and water. The total length of each nymph was measured and, the dry weight (24hs in an oven, $60^{\circ} \mathrm{C}$ ) was determined. The relation between total length $(\mathrm{mm})$ and dry weight $(\mathrm{mg})$ of each Telebasis nymph shaped an adjusted exponential curve, having as equation $y=0,31$ e $0,36 x(R 2=0,96)$. In conclusion, there was increase in the biomass of Telebasis in dry season due to great amount of food in this period. The biomass decreased when water temperature surface was lower. It is possible that the decrease of the water temperature influenced the emergence of the dragonflies adults and so we recorded only Odonate with lower biomass and juvenile..

Keywords: Biomass. Macrophytes. Odonata. Telebasis.

\section{INTRODUÇÃO}

Sample et al. (1) destacaram a importância de estudos envolvendo biomassa de insetos, dadas sua alta abundância e diversidade. Segundo Johnston e Cunjak (2), a biomassa pode ser uma ferramenta importante, por exemplo, em estudos sobre crescimento de macroinvertebrados como insetos aquáticos. A zona litorânea, especialmente junto às macrófitas, abriga a maior abundância e diversidade de insetos por proporcionar um habitat estável e local de refúgio contra predadores como Odonata $(3,4,5,6,7)$.

Odonata se destaca como um dos principais predadores da zona litorânea $(7,8)$. Sua dieta pode envolver desde invertebrados como Chironomidae (9) e cladóceros (10) até pós-larvas de peixes (11). Esta heterogeneidade alimentar é proporcional ao tamanho das ninfas de Odonata que pode variar de $8 \mathrm{~mm}$ em Protoneuridae a 62 mm em Aeshnidae (12).

Estudos sobre biomassa de Odonata são escassos (13). A grande dificuldade de criação e identificação dos estágios imaturos são as principais razões da falta de estudos com Odonata (12). Os autores ainda destacaram que a criação das ninfas é a única forma de identificação confiável em âmbito específico, entretanto, durante o processo de criação a mortalidade geralmente é muito elevada, podendo atingir 62,2\% em Telebasis (Selys, 1875).

Telebasis se destacou por sua alta abundância na Lagoa do Camargo, lateral ao Rio Paranapanema (14). Os objetivos deste trabalho foram estimar a variação da biomassa de ninfas de Telebasis durante treze meses, a partir de uma curva exponencial de ajuste.

\section{MATERIAIS E MÉTODOS}

As ninfas de Telebasis foram amostradas mensalmente, no período de março de 2004 a março de 2005, na Lagoa do Camargo, em três stands de E. azurea. Foi utilizado um puçá de formato quadrado (área $=0,16$ $\mathrm{m}^{2}$ ) com rede de $0,5 \mathrm{~mm}$ de malha. Em laboratório, a fauna foi removida com movimentos circulares da planta em baldes com formol 8\%, formol 4\% e água, consecutivamente (15). Os conteúdos dos baldes foram filtrados em peneira de $0,5 \mathrm{~mm}$ de malha e a fauna retida conservada em álcool $70 \%$. Numa fase inicial de triagem, com auxílio de um esteriomicroscópio Zeiss ${ }^{\circledR}$ mod. Stemi SV6, todas as ninfas de Telebasis (total de 29 indivíduos) foram isoladas dos demais taxa utilizando bibliografia específica e identificadas até gênero (12). As ninfas de Odonata foram identificadas até gênero, pois não houve sucesso na criação em laboratório dos imaturos amostrados em campo até se tornarem adultos e não foram encontrados adultos próximos aos sítios de amostragem. Os adultos de Odonata são fundamentais para identificação a nível específico. Apenas ninfas íntegras, ou seja, com todas as suas estruturas presentes, como cabeça, tórax, abdômen, patas, antenas e brânquias, tiveram seus comprimentos totais medidos, isto é, a partir da região anterior da cabeça até a porção final do $10^{\circ}$ segmento abdominal. Para a medição foi utilizado um sistema de imagem digital acoplado a um esteriomicroscópio Zeiss ${ }^{\circledR}$ mod. StemiSV6. Após a determinação do comprimento, os indivíduos de Telebasis foram levados em estufa a $60^{\circ} \mathrm{C}$ por 24 horas, para obtenção de seu peso seco. Após este período de 24 horas, as ninfas permaneceram em dessecador por uma hora e então pesadas em balança de precisão Mettler ${ }^{\circledR}$ mod. H20T (16). 


\section{RESULTADOS}

A média e o desvio padrão dos valores de biomassa em função das classes de tamanho estão registrados na Tabela 1.

No Gráfico 1 está registrada a relação entre peso seco $(\mathrm{mg})$ e comprimento total $(\mathrm{mm})$ de cada ninfa de Telebasis, assim como a curva exponencial de ajuste tendo como equação $y=0,31 \mathrm{e}^{0,36 \mathrm{x}} \mathrm{e} \mathrm{R}^{2}=$ 0,96 , onde $\mathrm{y}=$ peso seco $(\mathrm{mg})$ e $\mathrm{x}=$ comprimento total das ninfas ( $\mathrm{mm})$.

As maiores médias de comprimento total das ninfas de Telebasis foram registradas no período de agosto a outubro de 2004 e fevereiro de 2005 (Gráfico 2).

No Gráfico 3 estão representadas as médias \pm desvio padrão da biomassa (mg) de Telebasis durante o período estudado. Em março, novembro e dezembro de 2004 e janeiro e março de 2005 não registramos Telebasis. De agosto a outubro de 2004 e em fevereiro de 2005, registramos as maiores médias de biomassa de Telebasis, enquanto que as menores foram observadas no período de abril a julho de 2004.

As temperaturas mais altas foram registradas em abril, setembro e outubro de 2004 e janeiro de 2005 (Gráfico 4).

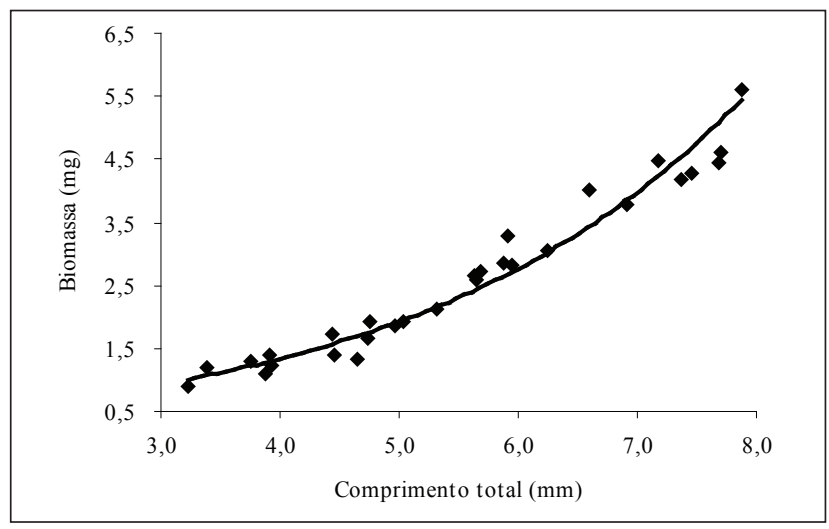

GRÁFICO 1 - Relação entre peso seco (mg) e o comprimento total $(\mathrm{mm})$ dos ninfas de Telebasis coletadas na Lagoa do Camargo, no período de março de 2004 a março de 2005

Fonte: Elaboração dos autores.
No Gráfico 5, podemos notar como foi a variação da precipitação mensal durante o período estudado. Ainda no Gráfico 5, pudemos definir as estações seca (abril a setembro de 2004) e chuvosa (outubro a março de 2005).

\section{DISCUSSÃO}

Os odonatos são obrigatoriamente carnívoros e vorazes predadores tanto na fase larval quanto na adulta $(17,18)$. Na estação seca é esperada maior abundância de macroinvertebrados graças à redução no nível da água. Afonso (15) observou que a abundância de macroinvertebrados junto à E. azurea foi maior nas lagoas laterais ao Rio Paranapanema na estação seca. Bispo e Oliveira (19) também notaram um aumento na abundância de macroinvertebrados na estação seca. Telebasis foi mais abundante também no período seco (14). Portanto, a abundância de Telebasis foi maior nos meses em que os demais macroinvertebrados foram mais abundantes, isto é, no período com maior oferta de alimento registramos maior abundância de predadores como ninfas de Telebasis. Além disso, notamos que a biomassa de Telebasis também foi maior na estação seca.

A biomassa de Telebasis foi mais alta nos dois últimos meses do período seco (agosto e setembro de 2004) e em dois meses da estação chuvosa (outubro de 2004 e janeiro de 2005). Durante a estação seca é esperada uma redução na biomassa de macroinvertebrados como Odonata, ou mesmo o desaparecimento de alguns taxa $(20,21)$. Todavia, registramos justamente o contrário, isto é, a biomassa de Telebasis foi maior no período seco em comparação ao chuvoso. Atribuímos este aumento na biomassa de Telebasis a um possível aumento na abundância e biomassa de outros macroinvertebrados como Chironomidae, uma de suas principais presas. Callisto et al. (22) observaram que Chironomidae apresentou maior biomassa na estação seca, assim como registramos para Telebasis.

TABELA 1 - Amplitude de variação, média \pm desvio padrão da biomassa (mg) em função das classes de tamanho (mm)

\begin{tabular}{cccc}
\hline Classes $(\mathbf{m m})$ & $\mathbf{n}$ & Média \pm DP & Amplitude de Variação (mg) \\
\hline 3 a 4 & 6 & $1,21 \pm 0,16$ & $0,9-1,4$ \\
4 a 5 & 5 & $1,60 \pm 0,25$ & $1,3-1,7$ \\
5 a 6 & 8 & $2,50 \pm 0,49$ & $1,9-3,3$ \\
6 a 7 & 4 & $3,41 \pm 0,57$ & $2,8-4,0$ \\
7 a 8 & 6 & $4,60 \pm 0,52$ & $4,2-5,6$ \\
\hline
\end{tabular}




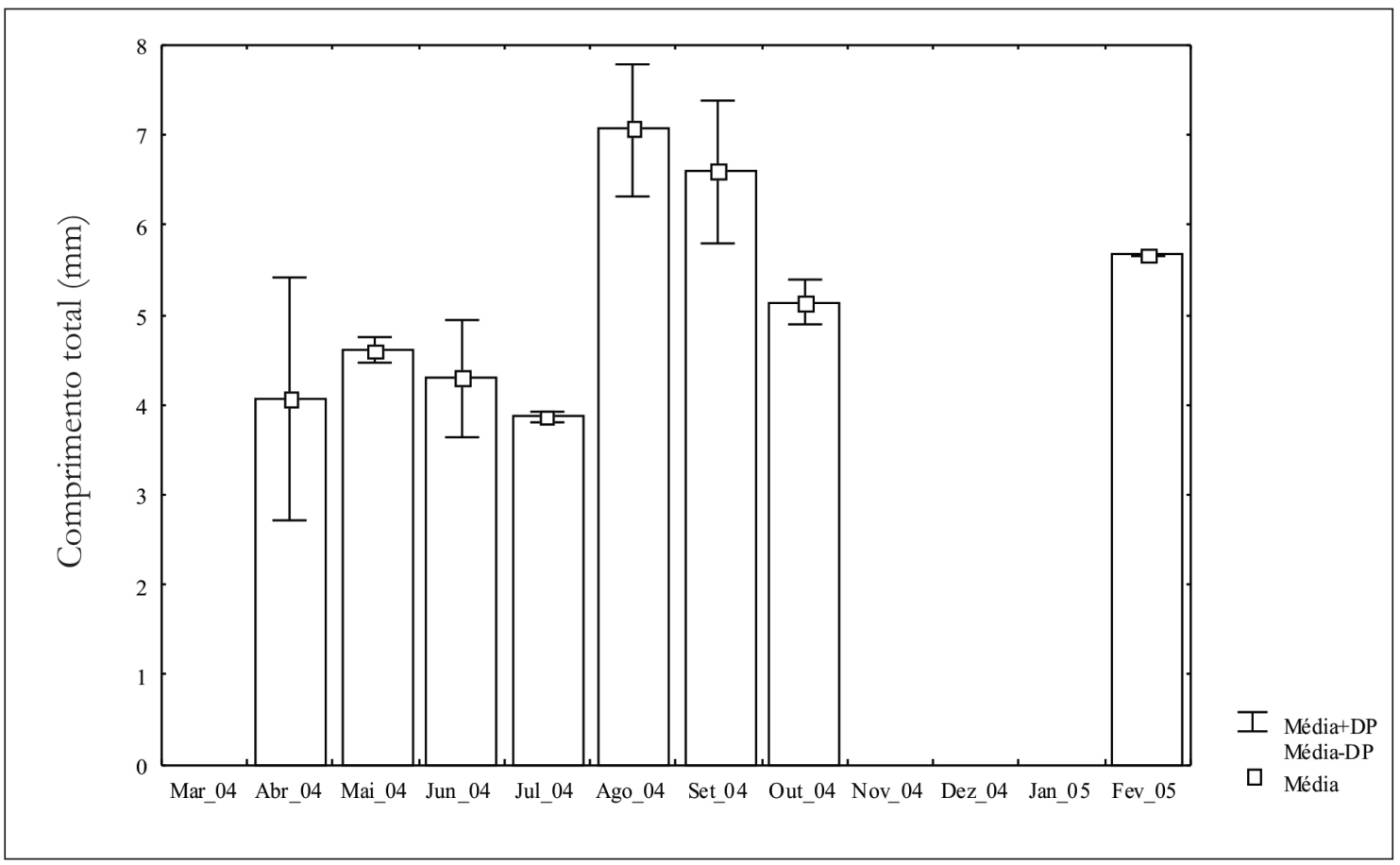

GRÁFICO 2 - Média \pm dp do comprimento total de ninfas de Telebasis amostrados de março de 2004 a março de 2005, na Lagoa do Camargo

Fonte: Elaboração dos autores.

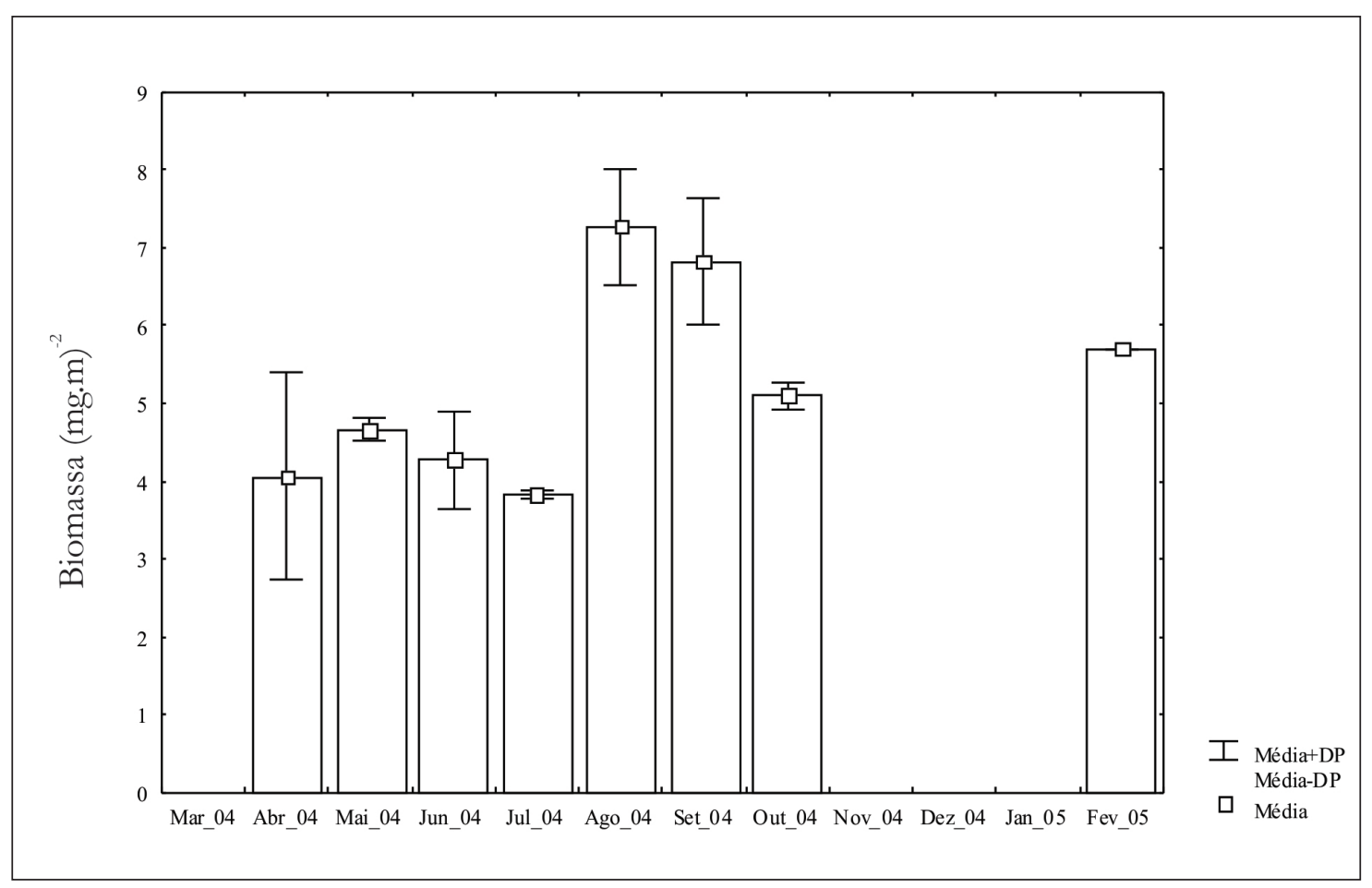

GRÁFICO 3 - Média \pm desvio padrão da biomassa de Telebasis amostrado de março de 2004 a março de 2005, na Lagoa do Camargo

Fonte: Elaboração dos autores. 


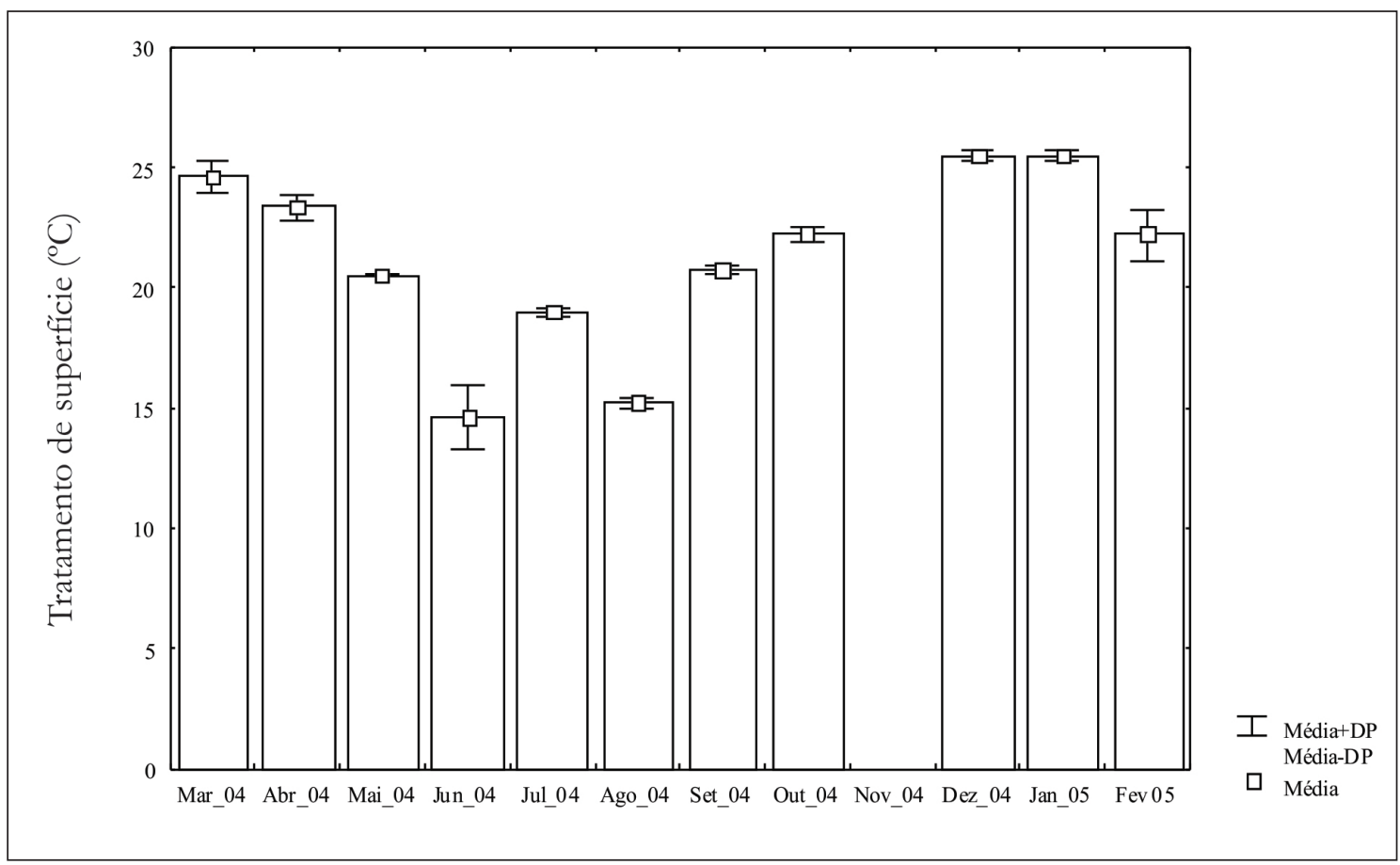

GRÁFICO 4 - Temperatura da água de superfície de março de 2004 a março de 2005, na Lagoa do Camargo

Fonte: Elaboração dos autores.

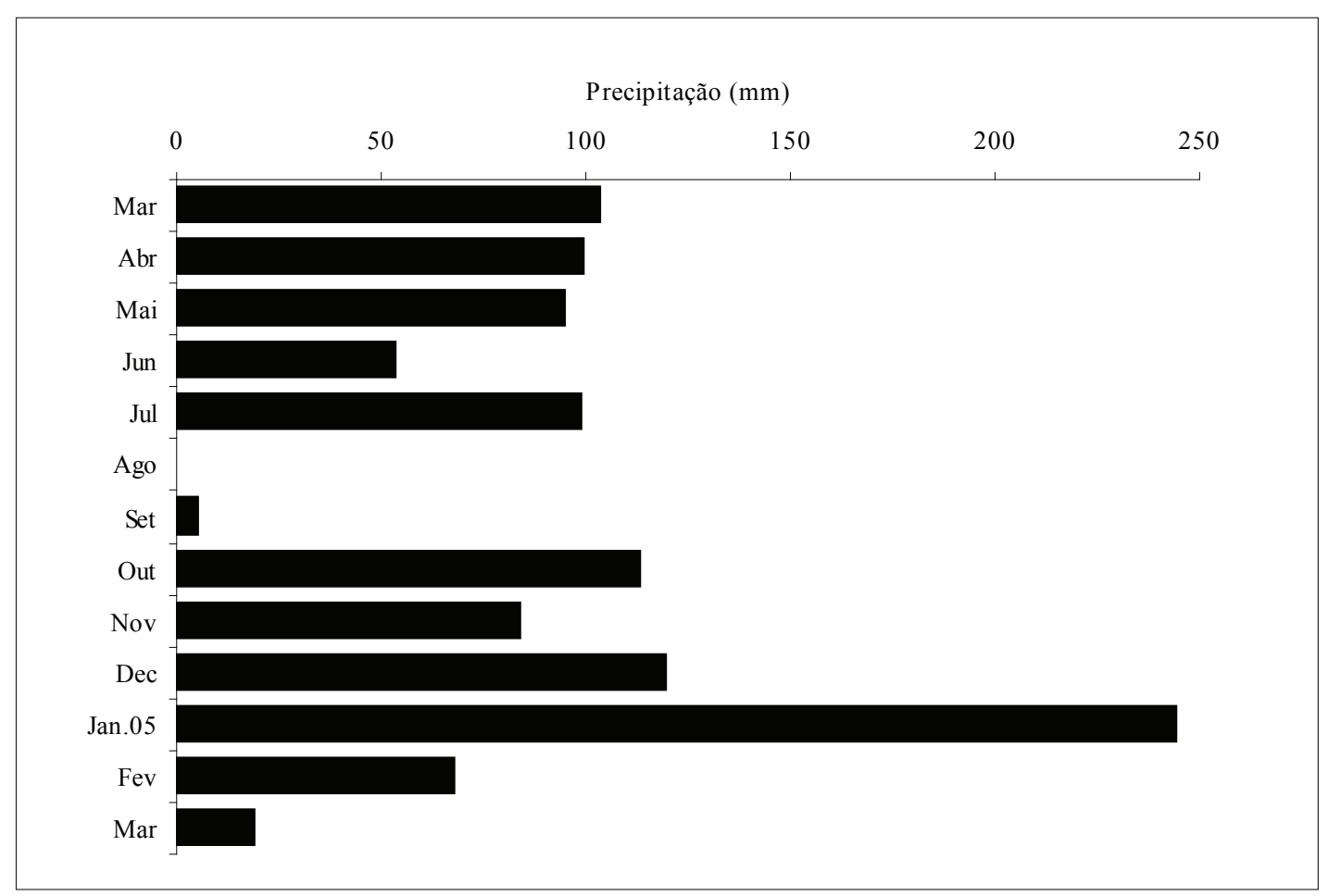

GRÁFICO 5 - Precipitação total mensal (mm) na Lagoa do Camargo, no período de março de 2004 a março de 2005

Fonte: Elaboração dos autores. 
Odonata se destaca dentre os insetos aquáticos como um dos principais predadores da zona litorânea $(7,8)$, sendo que os componentes mais abundantes de sua dieta são formas imaturas de Díptera como Chironomidae (9). Chironomidae também é, segundo Corbet (18), um dos principais insetos encontrados em conteúdo estomacal de Odonata. Portanto, era esperado que a variação na biomassa e abundância de Odonata fosse semelhante às biomassas e abundâncias de Chironomidae, uma de suas principais fontes de energia. Esta similaridade, principalmente com relação à biomassa de Odonata e Chironomidae, pode ser observada no período chuvoso. Callisto et al. (22) registraram redução de $58 \%$ na biomassa de Chironomidae no início do período chuvoso (setembro a outubro de 1993). No período de maior precipitação, também registramos $50 \%$ de decréscimo na biomassa de Telebasis, valores semelhantes aos registrados para Chironomidae.

Arruda (23) observou outra similaridade entre Odonata e Chironomidae. Segundo o autor, há correlação entre comprimento das ninfas de Odonata e biomassa de Chironomidae, ou seja, nos locais onde observou ninfas de Odonata de menor comprimento, registrou as maiores biomassas de Chironomidae. Infelizmente, não existem dados sobre biomassa de Chironomidae na Lagoa do Camargo. Entretanto, em junho, agosto e setembro registramos as maiores biomassas de Chironomidae (22). Neste período, segundo Arruda (23), poderíamos esperam as menores ninfas de Telebasis, porém só notamos esta correlação negativa entre comprimento de Odonata e biomassa de Chironomidae no mês de junho de 2004.

A biomassa pode ser uma ferramenta importante em estudos sobre crescimento de macroinvertebrados, como insetos aquáticos (2). Os autores observaram uma curva exponencial positiva entre a biomassa e o comprimento. Para Telebasis, também registramos curva exponencial positiva entre biomassa-comprimento, ou seja, as classes de tamanho menores apresentaram menor acúmulo de biomassa que as classes maiores.

No período de junho a agosto de 2004, observamos as menores biomassas e uma das menores médias de comprimento de Telebasis. Neste período, também registramos as menores temperaturas de superfície de água. Segundo Corbet (18), baixas temperaturas podem funcionar como estímulo para que ocorra emergência dos adultos de Odonata. Além disso, é comum em Odonata sincronismo de emergência, ou seja, as ninfas passam para a fase adulta em um curto espaço de tempo (18). Cothran e Thorp (24) também notaram o efeito direto da temperatura sobre a emergência dos adultos. Possivelmente nos meses de temperaturas mais amenas (junho a agosto), as ninfas em últimos instares e, portanto, com maior biomassa não foram encontradas talvez pela emergência das ninfas em adultos. Por esta razão, observamos apenas indivíduos jovens e, assim, com menor biomassa.

Em conclusão, houve um aumento na biomassa de Telebasis no período seco, em virtude do aumento na oferta de alimento provocado pela redução no nível da água. A biomassa foi menor nos meses mais frios, possivelmente pela emergência dos adultos, sendo registrados apenas indivíduos jovens e com menor biomassa.

\section{REFERÊNCIAS}

1. Sample BE, Cooper RJ, Greer RD, Whitmore RC. Estimation of insect biomass by length and width. American Midland Naturalist. 1993;129(2):234-40.

2. Johnston TA, Cunjak RA. Dry mass-length relationships for bentic insects: a review with new data from Catamaran Brook, New Brunswick, Canada. Freshwater Biology. 1999;41:653-74.

3. Ward JV. Aquatic insect ecology. New York: John Wiley \& Sons; 1992.

4. Humphries P. Aquatic Macrophytes, macroinvertebrate associations and water levels in a lowland Tasmanian river. Hydrobiologia. 1996;321:219-33.

5. Stewart DAB, Samways MJ. Conserving dragonfly (Odonata) assemblages relative to river dynamics in an African Savanna Game Reserve. Conservation Biology. 1998;12(3):683-91.

6. Ferreira-Peruquetti PS, De Marco P. Efeito da alteração ambiental sobre a comunidade de Odonata em riachos de Mata Atlântica de Minas Gerais, Brasil. Rev Bras de Zoolog. 2002;19(2):317-27.

7. Capitulo AR. Los Odonata de la República Argentina (Insecta). La Plata: Profadu (Conicet); 1992.

8. Costa C, Ide S, Simonka CE. Insetos ninfas: metamorfose e identificação. Ribeirão Preto: Holos; 2006.

9. Buttow NC, Takeda AM, Melo SM. Fauna de Odonata da planície de inundacão do Alto Rio Paraná: identificacão e ocorrência de náiades. UNIMAR. 1993;15(Suplemento):83-94. 
10. Soares CM, Hayashi C, Faria ACEA. Influência da disponibilidade de presas, do contraste visual e do tamanho das larvas de Pantala sp. (Odonata, Insecta) sobre a predação de Simocephalus cerrulatus (Cladocera, Crustacea). Acta Scientiarum. 2001;23(2):357-62.

11. Soares CM, Hayashi C, Reidel A. Predação de pós-larvas de curimba (Prochilodus lineatus, Valenciennes, 1836) por larvas de Odonata (Pantala, Fabricius, 1798) em diferentes tamanhos. Acta Scientiarum. 2003;25(1):95-100.

12. Costa JM, Souza LOI, Lourenço AN, Oldrini BB. Chave para identificação das famílias e gêneros das larvas conhecidas de Odonata do Brasil: comentários e registros bibliográficos. Publicação Avulsa Museu Nacional. 2004;99:3-42.

13. Runk C, Blinn DW. Secondary production by Telebasis salva (Odonata) in a thermally constant aquatic ecosystem. Journal of the North American Benthological Society. 1993;12(2):136-47.

14. Fulan JA, Henry, R. The Odonata (Insecta) assemblage on Eichhornia azurea (Sw.) Kunth (Pontederiaceae) stands in Camargo Lake, a lateral lake on the Paranapanema River (state of São Paulo, Brazil), after an extreme inundation episode. Acta Limnologica Brasiliensia. 2006;18(4):423-31.

15. Afonso AAO. Relações da fauna associada à Eichhornia azurea (Swartz) Kunth com as variáveis abióticas em lagoas laterais de diferentes graus de conexão ao Rio Paranapanema (zona de desembocadura na Represa de Jurumirim, SP). [tese]. Botucatu: Universidade Estadual Paulista Júlio de Mesquita Filho; 2002.

16. Mccauley E. The estimation of the abundance and biomass of zooplankton in samples. In: Downing JA, Ringler FH, editores. A manual on methods for the assessment of secondary productivity in fresh waters. Oxford, Blackwell Scientific Publications; 1984. p. 228-65.

17. Carvalho AL, Nessimian JL. Odonata do Estado do Rio de Janeiro, Brasil: hábitats e hábitos das larvas. In: Nessimian JL, Carvalho AL, editores. Ecologia de Insetos Aquáticos. Rio de Janeiro: Series Oecologia Brasiliensis; 1998. p. 3-28.

18. Corbet PS. Dragonflies: Behavior and Ecology. New York Cornell: University Press; 1999.
19. Bispo PC, Oliveira LG. Distribuição espacial de insetos aquáticos (Ephemeroptera, Plecoptera e Trichoptera) em córregos de cerrado do Parque Ecológico de Goiânia, Estado de Goiás. In: Nessimian JL, Carvalho AL, editores. Ecologia de Insetos Aquáticos. Rio de Janeiro, Series Oecologia Brasiliensis; 1998. p. 175-189.

20. Batzer DP, Wissinger SA. Ecology of insect communities in non-tidal wetlands. Annual Review of Entomology. 1996;41:75-100.

21. Wellborn GA, Skelly DK, Werner EE. Mechanisms creating community structure across a freshwater habitat gradient. Annual Review of Ecology and Systematics. 1996;27:337-63.

22. Callisto M, Moreno P, Gonçalves JFJr, Leal JJF, Esteves FA. Diversity and biomass of Chironomidae (Díptera) larvae in an impacted coastal lagoon in Rio de Janeiro, Brazil. Brazilian Journal Biology. 2002;62(1):77-84.

23. Arruda JA. A consideration of trophic dynamics in some tallgrass prairie farm ponds. American Midland Naturalist, Manhattan. 1979;102(2):254-62.

24. Cothran ML, Thorp JH. Emergence patterns and size variation of Odonata in a Thermal Reservoir. Freshwater Invertebrate Biology. 1982;1(4):30-39.

Recebido: 06/04/2007 Received: 04/06/2007

Aprovado: 22/12/2007 Approved: 12/22/2007 